\title{
GEOTECHNICAL HAZARD REPRESENTATION FOR SEISMIC RISK ANALYSIS
}

\author{
Sonia Giovinazzi ${ }^{1}$
}

\begin{abstract}
SUMMARY
Seismic risk analysis, either deterministic or probabilistic, along with the use of a GIS-environment to represent the results, are helpful tools to support decision making for planning and prioritizing seismic risk management strategies. This paper focuses on the importance of an appropriate geotechnical hazard representation within a seismic risk analysis process.
\end{abstract}

An overview of alternative methods for geotechnical zonation available in literature is provided, with a level of refinement appropriate to the information available. It is worth noting that in such methods, the definition of the site effect amplifications does not account for the characteristics of the built environment affecting the soil-structure interaction. Alternative methods able to account for both the soil conditions and the characteristics of the built environment have been recently proposed and are herein discussed.

Within a framework for seismic risk analysis, different formulations would thus derive depending on both the intensity measure and the vulnerability approach adopted. In conclusion, an immediate visualization of the importance of the geotechnical hazard evaluation within a seismic risk analysis is provided in terms of the variation of the expected damage and consequence distribution with reference to a case-study.

\section{INTRODUCTION}

The aim of a seismic risk is the estimation and the hypothetical, quantitative description of the consequences of a seismic event upon a geographical area (a city, a region, a state or a nation) in a certain period of time (where probabilistic methods can be viewed as inclusive of all possible deterministic events with a finite probability of occurrence). The effects to be predicted are the physical damage to buildings and other facilities, the number of casualties, the potential economic losses due to the direct or indirect costs, including business interruption and downtime, the loss of function in lifelines and critical facilities, as well as the impacts at the social, organizational and institutional levels.

The results provided by seismic risk analysis, either probabilistic or deterministic, could thus be regarded as helpful guidelines during all the phases of risk management, before and after the critical event. It is worth noting that, the choice between deterministic or probabilistic risk analysis depends on the aims of the study. When prevention measures at a territorial scale are of interest, a probabilistic risk analysis is preferable, in that it brings together the effects of all the potential seismic sources of the area and supplies a comparable evaluation between all the different communities interested by the study. On the other hand, when issues related to emergency management are of interest, a deterministic analysis, commonly referred to as a scenario analysis (simulating a representative earthquake) is the most meaningful, in that it reproduces a realistic distribution of the effects on the territory.

The common framework of both probabilistic and deterministic seismic risk analysis is based on the traditionally accepted definition of seismic risk as the convolution of hazard, exposure and vulnerability. The hazard analysis aims to characterize the seismic motion expected in the region, in terms of physical measures or in terms of macroseimic intensity, possibly including the local amplifications (microzoning). It is well established that local site conditions and, to a more limited extend, irregular surface topography can substantially influence the amplitude, the frequency content and the duration of a strong ground motion and consequently can exert a crucial influence on the severity of the damage caused by the earthquake on the single structure. Similarly, when considering a territorial scale seismic risk analyses, regardless of the approach used for the estimation of the seismic hazard and of the intensity measure parameter adopted, the influence of site conditions cannot be disregarded. The exposure analysis aims to evaluate the number and characteristics of the built environment in a given area, both in quantitative and qualitative terms, while the vulnerability analysis aims to estimate the intrinsic likelihood of the structures to be damaged due to an earthquake motion by correlating the severity of the seismic motion with the expected structural and non-structural damage. By convolution of the seismic hazard with the vulnerability and exposure, an estimation of the distribution of damage, of the related economical losses and of the consequences to buildings and people can be carried out.

In this paper the attention will be focused on the effects of alternative methodology and level of geotechnical zonation on the final results of a deterministic seismic risk analysis, with reference to site effect amplifications due to soil and morphological condition. After an overview of alternative geotechnical zonation methods, differently defined depending on the available level of knowledge/information, it is discussed how to account for site effects within a seismic risk analysis. In particular, reference is made to the seismic motion representation in terms of macroseismic intensity where the building vulnerability is assessed according to a macroseimic approach [1]. The influence of accounting for the actual soil conditions within a seismic risk analysis is presented in terms of variation of the resulting probabilities of expected damage levels and expected consequences with reference to a studycase.

\footnotetext{
${ }^{1}$ Research Fellow, University of Canterbury, Christchurch, New Zealand (Member).
} 


\section{REPRESENTATION OF ALTERNATIVE SOURCES OF GEOTECHNICAL HAZARDS WITHIN A SEISMIC RISK ANALYSIS}

\subsection{Local ground motion amplification due to soil conditions}

As mentioned, a scenario study aims at estimating the level and distribution of damage at a territorial scale, instead of predicting the response of a specific structure at a specific site. When the scope is to generate a geotechnical zonation to be employed for vulnerability assessment and seismic risk purposes, the representation of the ground conditions, needs to be no more detailed than that required by design seismic code provisions. Furthermore, simplified approaches for predicting the ground and the structural response at specific sites can actually be implemented.

In order to map out geological units associated to local ground motion amplification, the Manual for Zonation on Seismic Geotechnical Hazards, TC4-ISSMGE [2] suggests, for example, three different levels of methodologies, depending on the level of available data on the soil site characteristics. A basic, "grade I", zonation level can be achieved by the compilation and interpretation of existing information available from historic documents (i.e. compiled data on the distribution of damage induced during past destructive earthquake), published reports and other available databases or by direct reference to the site surface geology. A more refined, "grade II", zonation level, comprises of additional sources of data obtainable at moderate cost. A very high and detailed zonation level, referred to as "grade III", typical of site and structural specific studies, is instead judged not to feasible and affordable for investigation on large areas. Once the geotechnical zonation is defined, TC4 manual [2] proposes different methods to account for the local ground motion amplification depending on the parameters employed for the hazard description. When the expected hazard is represented in terms of macroseimic intensity, empirical correlations between the surface geology and the increment of the seismic intensity, based on post-event observations, are proposed. Table 1 and Table 2 show, as an example, the intensity increments proposed respectively by Medvedev [3] and Everdnden and Thomson [4]. Alternatively, relative amplification factors, $f_{a g}$ related to surface geology are suggested by Midorikawa [5], to be adopted when the hazard is represented in terms of peak ground acceleration or spectral ordinates (Table 3 ). The relative amplification factors, $\mathrm{f}_{\mathrm{ag}}$ have been translated in terms on increments of macroseismic intensity, implementing Equation 4 (Tab. 3).

Table 1. Intensity increments $\Delta \mathrm{I}$ for geology units after Medvedev [3] in TC4-ISSMGE[2].

\begin{tabular}{lc}
\hline \multicolumn{1}{c}{ Medvedev [3] } & $\Delta \mathbf{I}_{\text {M.M.I }}$ \\
\hline Granites & 0 \\
Limestone, Sandstone, Shale & $0.2-1.3$ \\
Gypsum, Marl & $0.6-1.4$ \\
Coarse-material ground & $1-1.6$ \\
Sandy Ground & $1.2-1.8$ \\
Clayey Ground & $1.2-2.1$ \\
Fill & $2.3-3$ \\
Moist ground (gravel, sand, clay) & $1.7-2.8$ \\
Moist fill and soil ground & $3.3-3.9$ \\
\hline
\end{tabular}

Table 2. Intensity increments $\Delta I$ for geology units after Everdnden and Thomson [4] in TC4-ISSMGE [2].

\begin{tabular}{lc}
\hline Everdnden and Thomson [4] & $\Delta \mathbf{I}_{\text {M.M.I }}$ \\
\hline Granitic \& metamorphic rocks & 0 \\
Paleozoic Rock & 0.4 \\
Early Mesozoic rocks & 0.8 \\
Cretaceous to Eocene rocks & 1.2 \\
Undivided Tertiary rocks & 1.3 \\
Oligocene to middle pliocene rocks & 1.5 \\
Pliocene-Pleistocene rocks & 2 \\
Tertiary volcanic rocks & 0.3 \\
Quaternary volcanic rocks & 0.3 \\
Alluvium (water table $<30 \mathrm{ft})$ & 3 \\
Alluvium $(30 \mathrm{ft}<$ water table $<100 \mathrm{ft})$ & 2 \\
Alluvium $(100 \mathrm{ft}<$ water table) & 1.5 \\
\hline
\end{tabular}

Table 3. Relative amplification factors, $\mathbf{f}_{a g}$ after Midorikawa [5] in TC4-ISSMGE [2].

\begin{tabular}{lcc}
\hline \multicolumn{1}{c}{ Midorikawa (1987) } & $\mathbf{f}_{\text {ag }}$ & $\Delta \mathbf{I}_{\text {M.M.I }}$ \\
\hline Holocene & 3.0 & 2.3 \\
Pleistocene & 2.1 & 1.6 \\
Quaternary volcanic rocks & 1.6 & 1.0 \\
Miocene & 1.5 & 0.9 \\
Pre-Tertiary & 1.0 & 0 \\
\hline
\end{tabular}

On a similar trend, the handbook for earthquake ground motion scenarios Faccioli and Pessina [6] prepared within the framework of the European project Risk-UE [7] distinguishes between two different levels of approaches depending on the data and information available as well as on the scope of the scenario study. In particular, a "level I" zonation can be obtained by the interpretation of the near-surface formations from the geological map in terms of approximate geotechnical units, using available geotechnical parameters, or some seismic response measure. A "level II" approach requires, instead, that as much data as possible on the subsoil are collected from public and private sources. Useful data for the latter level could be given by soil borings, water wells, field geophysical investigations, geotechnical laboratory tests and geotechnical borings, especially when reaching formations regarded as "seismic bedrock". The collected data have to be careful selected, assembled and processed according to different steps that allow to draw contours of the shear wave velocity in the uppermost $30 \mathrm{~m}\left(\mathrm{Vs}_{30}\right)$, throughout the analysed area. The approximate geotechnical units defined either according to a level I approach and to the $\mathrm{Vs}_{30}$ contours resulting from the level II approach can then be re-arranged according to the typical soil classifications adopted in code standards. According to the handbook for earthquake ground motion scenarios [6], the local ground motion amplification can be accounted for as follows, depending on the parameters employed for the hazard description: a) when the expected hazard is represented in terms of macroseimic intensity, an increment of 0.5 intensity degree $(\Delta \mathrm{I}=0.5)$ is suggested for medium stiff clays and medium dense cohesionless soils when compared to the stiff soils and rock benchmark, in line with what suggested by Bard [8]; b) when the hazard is represented in terms of physical parameters, elastic response spectra $\mathrm{S}_{\mathrm{ae}}(\mathrm{T})$ derived from code provisions or predictive equations, have to be directly related to classes of soil. 
Table 4. Soil classes, range of shear wave velocity $\mathrm{Vs}_{30}$ according to EC8 [9] and corresponding Intensity increments $\Delta I$ (according to Faccioli and Pessina [6]).

\begin{tabular}{|c|c|c|c|}
\hline $\begin{array}{l}\text { Soil } \\
\text { Class }\end{array}$ & $\begin{array}{c}\text { Description of stratigraphic } \\
\text { profile }\end{array}$ & $\begin{array}{l}V_{\text {S30 }} \\
(\mathrm{m} / \mathrm{s})\end{array}$ & $\Delta \mathbf{I}_{\text {EMS98 }}$ \\
\hline A & $\begin{array}{l}\text { Rock or other rock-like } \\
\text { geological formation, } \\
\text { including at most } 5 \mathrm{~m} \text { of } \\
\text { weaker material at the surface }\end{array}$ & $>800$ & 0 \\
\hline B & $\begin{array}{l}\text { Deposits of very dense sand, } \\
\text { gravel, or very stiff clay, at } \\
\text { least several tens of } m \text { in } \\
\text { thickness, characterised by a } \\
\text { gradual increase of mechanical } \\
\text { properties with depth }\end{array}$ & $360-800$ & 0 \\
\hline $\mathrm{C}$ & $\begin{array}{l}\text { Deep deposits of dense or } \\
\text { medium - dense sand, gravel } \\
\text { or stiff clay with thickness } \\
\text { from several tens to many } \\
\text { hundreds of } m\end{array}$ & $180-360$ & 0.5 \\
\hline D & $\begin{array}{l}\text { Deposits of loose-to-medium } \\
\text { cohesionless soil (with or } \\
\text { without some soft cohesive } \\
\text { layers), or of predominantly } \\
\text { soft-to-firm cohesive soil }\end{array}$ & $<180$ & 1 \\
\hline
\end{tabular}

\subsection{Local ground motion amplification due to surface irregularities}

A further main source of local amplification is due to surface irregularities. Amplification factors, $f_{a g}$ are available in code provisions when the seismic intensity is described in terms of peak ground acceleration or spectral ordinates. In the Eurocode EC8 factors $f_{a g}$ in the range of $1.2 \div 1.4$ have been derived from numerical simulations on different irregular profiles. Results of refined numerical 2D and 3D simulations of the expected ground motion amplification performed by Paolucci and Rimordi [10], in the real case of four steep topographic configurations provided satisfactory confirmations of these values (Table 5).

Table 5. Topographic amplification factors by EC8 [9] and Paolucci et Rimardi [10] for different site morphology and corresponding intensity increments $\Delta \mathrm{I}[6]$.

\begin{tabular}{|c|c|c|c|c|c|}
\hline \multirow{2}{*}{ Site morphology } & \multicolumn{4}{|c|}{ Amplification factors } & \multirow{2}{*}{$\Delta \mathbf{I}$} \\
\hline & EC8 & 3D & 2D SH & 2D SV & \\
\hline Isolated Cliff & 1.2 & 1.3 & 1.22 & 1.22 & 1 \\
\hline $\begin{array}{l}\text { Ridge crest width } \\
<<\text { base width } \\
\text { average slope angle } \\
>30^{\circ}\end{array}$ & 1.4 & 1.58 & 1.18 & 1.32 & 1.5 \\
\hline $\begin{array}{l}\text { Ridge crest width } \\
<<\text { base width } \\
\text { average slope angle } \\
<30^{\circ}\end{array}$ & 1.2 & 1.25 & 1.09 & 1.28 & 1 \\
\hline
\end{tabular}

In order to empirically derive intensity increments $\Delta \mathrm{I}$ for an hazard assessment in terms of macroseimic intensity, the evidence of topographic amplification have to be found within available data from historical earthquakes. Based on the macroseismic observations of the 1887 Western Liguria earthquake event, Faccioli et al. [11] have established that within an epicentre distance of few tenths of kilometres, amplification on markedly irregular topography (i.e. hilltop, crests and severely sloping ground) can in general lead to an intensity increment $\Delta \mathrm{I}=1$ and, only exceptionally, to $\Delta \mathrm{I}=1.5$ or more (Figure $4 \mathrm{a})$.

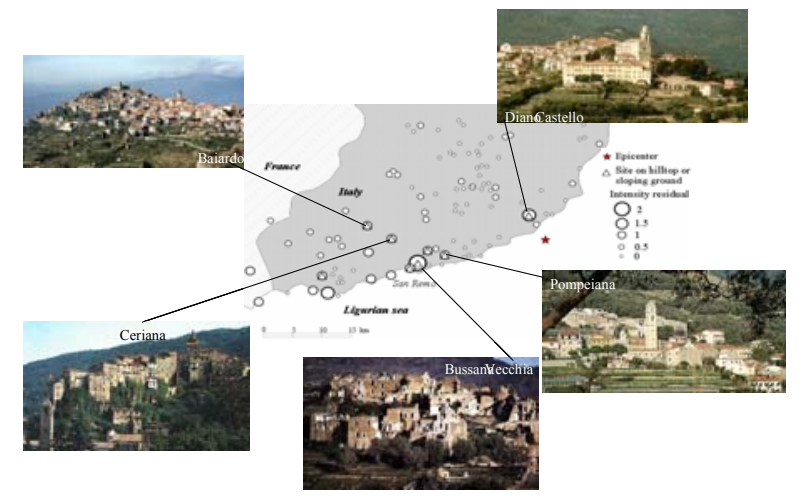

Figure 1:

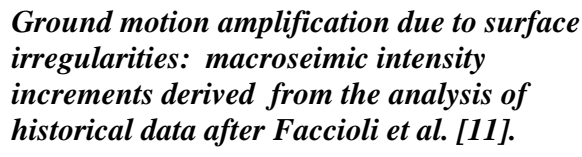

\subsection{Earthquake-induced geotechnical hazard}

The impact of induced hazard, such as significant soil permanent deformations, rock falls and landslides, ground settlements and ruptures due to liquefaction and lateral soil spreading, has to be accounted for within a seismic risk analysis. Due to the diversity and complexity of such phenomena, further developments are in general still required for the definition of accurate while simplified methods to be implemented in a seismic risk analysis framework.

A first step in the investigation would consist of searching for historical evidences of earthquake induced liquefaction and landsliding phenomena within, or in the vicinity of an analyzed area [6]. In addition, loose, water saturated artificial fills in correspondence of old river (or creek) beds, characterized by the presence of 3-5 $\mathrm{m}$ or more of soft soil, should be accurately mapped (also according to the EC8) as highly vulnerable sites for permanent ground deformation and soil failures. On the other hand, highly vulnerable sites for seismically trigger landslides are the ones where slopes are predominantly subject to frictional types of failures. Examples of simplified procedures for the evaluation of earthquakeinduced landslides and liquefaction phenomena within seismic risk analysis can be found in Siyahi and Ansal [12], within TC4-ISSMGE [2], and Bird et al. [13], respectively. An overview of alternative methods for the representation of earthquake-induced geotechnical hazards due to liquefaction for implementation within seismic risk analysis and the proposal of more refined approaches can be found in Giovinazzi and Cubrinovski [14]. It is worth highlighting that that in this paper the focus has been on site amplification due to soil and surface irregularities while the representation and of earthquake induced hazards have not been further investigated.

\section{FORMULATION OF PERIOD-DEPENDENT SITE EFFECT AMPLIFICATIONS}

\subsection{Period dependent site effect amplifications for a mechanical-based vulnerability method}

When a mechanical-based approach is adopted for the vulnerability assessment, such as the capacity spectrum method, implemented within HAZUS [16] and Risk-UE project [7], the hazard is described in terms of elastic response 
spectra $\mathrm{S}_{\mathrm{ae}}(\mathrm{T})$ for different classes of soil, thus directly allowing for the evaluation of period-dependent site effects.

As mentioned in the previous paragraph, both soil conditions and morphological properties can be taken into account directly by assuming and properly modifying pre-defined spectral shapes. However, when elastic response spectra need to be derived by predictive equations (attenuation laws), only a discrete numbers of the fundamental period are available. In the latter case, as discussed in Giovinazzi [16], the definition of the characteristic period $\mathrm{T}_{\mathrm{C}}$, which defines the starting point of the decay of the spectral acceleration ordinates, can be of significant importance when implementing a closed-form solution for the evaluation of the performance point. In this case, the definition of the characteristic period $\mathrm{T}_{\mathrm{C}}$ is subjected to various proposals, i.e HAZUS [15] and Giovinazzi [16].

\subsection{Period-dependent site effect amplifications for macroseismic vulnerability method}

On the other hand, when the seismic hazard is represented in terms of macroseismic intensity, soil amplifications can be taken into account by increasing, locally, the intensity, I, evaluated on the bed-rock, as discussed in Par. 2.1. However, such approaches do not account for the differences in the dynamic amplification related to the fundamental frequencies of both the soil and the structure, nor due to more complex soil structure interaction effects.

In order to overcome these limitations, an alternative method has been proposed by Giovinazzi and Lagomarsino [1] where macroseismic intensity increments are derived as a function of relative amplification factors, $\mathrm{f}_{\mathrm{ag}}$ defined for different building types and soil condition. Referring to a predefined spectral shape provided by seismic code prescriptions, the factor $\mathrm{f}_{\mathrm{ag}}$ is evaluated as the ratio between the elastic response spectrum $\mathrm{S}_{\mathrm{ae}}$ evaluated for the fundamental period $\mathrm{T}$ for a certain soil class $\mathrm{k}, \mathrm{S}_{\mathrm{ae}}(\mathrm{T})_{\mathrm{k}}$, and the elastic response spectrum $\mathrm{S}_{\mathrm{ae}}$ evaluated for the fundamental period $\mathrm{T}$ and for rock conditions, $\mathrm{S}_{\mathrm{ae}}(\mathrm{T})_{\mathrm{A}}$, (Eq. 1).

$$
f_{a g}=\frac{S_{a e}(T)_{k}}{S_{a e}(T)_{A}}
$$

An appropriate estimation of the elastic period of the structures is thus required as a first step. In this contribution, the evaluation of peak ground acceleration amplification factors have been derived for different type of buildings, i.e. unreinforced masonry, URM, pre-1970s reinforced concrete, $\mathrm{RCp}$, and reinforced concrete buildings designed according to more recent seismic code previsions, RC are distinguished. For each building typology the fundamental period $\mathrm{T}$ has been evaluated according to the following Eq. 2:

$$
\mathrm{T}=\alpha \mathrm{H}^{\beta}
$$

where $\mathrm{H}$ is the building height, evaluated assuming: an average number of floors $\mathrm{N}$ as representative of low ( $L$ ), medium ( $(\mathrm{M})$ and high-rise $(\mathrm{H})$ buildings $(\mathrm{N}=2,4,6$ and $\mathrm{N}$ $=3,5,8$ respectively for masonry and reinforced concrete types), a characteristic inter-storey-drift for each typology ( $\mathrm{h}=$ $3 \mathrm{~m}$ for masonry types, $\mathrm{h}=3.5 \mathrm{~m}$ for reinforced concrete ones), $\alpha$ and $\beta$ are coefficients differently specified depending on the building typology. In particular, $\alpha=0.05, \beta=0.75$ for masonry types and $\alpha=0.075 \beta=0.75$ for well-designed r.c. buildings according to EC 8 prescriptions, while $\alpha=0.065$ and $\beta=0.9$ for reinforced concrete buildings designed prior to the introduction of modern seismic prescriptions (in the mid-1970 in most seismic prone countries), in order to accounting for the extensive cracking of the structural members and thus obtain a conservative estimation of the building displacement demand [17].

The so derived $f_{a g}$ factors can be also translated in terms of intensity increments $\Delta \mathrm{I}$ by assuming a correlation between the macroseismic intensity $I$ and the peak ground acceleration $a_{g}$ $\left(\mathrm{I}-\mathrm{a}_{\mathrm{g}}\right)$. A generalized expression of the $\mathrm{I}-\mathrm{a}_{\mathrm{g}}$ correlations, able to fit most of the relationships proposed in literature, can be suggested in the form of:

$$
\mathrm{a}_{\mathrm{g}}=\mathrm{c}_{1} \mathrm{c}_{2}{ }^{\left(\mathrm{I}-\mathrm{I}_{\mathrm{o}}\right)}
$$

where $c_{1}$ represents the peak ground acceleration value $a_{g}$ corresponding to the reference intensity $I_{o}$ and $c_{2}$ measures the rate of increase of the peak ground acceleration ag with the intensity I. Given the expression above, the intensity increment $\Delta \mathrm{I}$ corresponding to the $\mathrm{f}_{\mathrm{ag}}$, derived according to Eq. 1, can thus be evaluated as

$$
\Delta \mathrm{I}=\frac{\ln \mathrm{fa}_{\mathrm{g}}}{\ln \mathrm{c}_{2}}
$$

Table 6 shows the $\mathrm{f}_{\mathrm{ag}}$ factors referred to the EC8 spectral shape for a magnitude Ms greater than 5.5, assuming $c_{1}=0.03$, $c_{2}=1.6$ for the $\mathrm{I}-\mathrm{a}_{\mathrm{g}}$ correlation (found to be a reliable I- $\mathrm{a}_{\mathrm{g}}$ correlation for the European territory as explained in Giovinazzi [16]. Table 7 shows the macroseismic intensity increment. $\Delta \mathrm{I}$, corresponding to the relative amplification factors $f_{a g}$ in Table 6. Alternatively, predictive equations (law attenuation) can be used. Table 8 shows the $f_{a g}$ factors and the corresponding $\Delta \mathrm{I}$ increments when using the predictive equations (attenuation laws) proposed by Ambraseys et al. [18], which provide acceleration response ordinates for discrete values of the fundamental period $\mathrm{T}$ and refers to four soil classes almost coincident with the EC8 classification. It is worth noting that the $f_{\text {ag }}$ factors and the $\Delta \mathrm{I}$ increments are invariable for different values of the magnitude $M$ and of the site-source distances R.

When comparing the $\mathrm{f}_{\text {ag }}$ values evaluated according to the two methods, it can be noted that the latter ones are lower and in general less sensitive to the fundamental period $\mathrm{T}$ of the structure type. On the other hand, both peak ground acceleration factors $f_{\text {ag }}$ and macroseimic intensity increments $\Delta \mathrm{I}$ are consistent with those proposed by Everdnden and Thomson [4] and shown in Tab. 2 as well as with the amplification factors defined by Midorikawa [5] and suggested by TC4-ISSMGE [2].

Table 6. $f_{\text {ag }}$ factors evaluated according to EC8 Type 1 spectrum (Ms $>5.5$ ), for the different soil classes and for different building categories.

\begin{tabular}{lllccccc}
\hline \multirow{2}{*}{ Building Types } & $\mathbf{N}$ & $\mathbf{h}[\mathbf{m}]$ & $\mathbf{T}[\mathbf{s}]$ & \multicolumn{5}{c}{$\mathbf{f}_{\text {ag }}$} \\
& & & & $\mathbf{B}$ & $\mathbf{C}$ & $\mathbf{D}$ & $\mathbf{E}$ \\
\hline URM_L & 2 & 3 & 0.19 & 1.20 & 1.15 & 1.35 & 1.40 \\
URM_M & 4 & 3 & 0.32 & 1.20 & 1.15 & 1.35 & 1.40 \\
URM_H & 6 & 3 & 0.44 & 1.31 & 1.26 & 1.47 & 1.53 \\
RC_L & 3 & 3.5 & 0.44 & 1.31 & 1.26 & 1.48 & 1.53 \\
RC_M & 5 & 3.5 & 0.64 & 1.50 & 1.73 & 2.17 & 1.75 \\
RC_H & 8 & 3.5 & 0.91 & 1.50 & 1.73 & 2.70 & 1.75 \\
RCp_L & 3 & 3.5 & 0.54 & 1.50 & 1.55 & 1.82 & 1.75 \\
RCp_M & 5 & 3.5 & 0.85 & 1.50 & 1.73 & 2.70 & 1.75 \\
RCp_H & 8 & 3.5 & 1.30 & 1.50 & 1.73 & 2.70 & 1.75 \\
\hline
\end{tabular}


Table 7. Macroseimic intensity increments $\Delta \mathrm{I}$ evaluated according to EC8 Type 1 spectrum (Ms $>5.5$ ), for the different soil classes and for different building categories.

\begin{tabular}{lcccc}
\hline \multirow{2}{*}{ Building Types } & \multicolumn{4}{c}{$\Delta \mathbf{I}$} \\
& B & C & D & E \\
\hline URM_L & 0.4 & 0.3 & 0.6 & 0.7 \\
URM_M & 0.4 & 0.3 & 0.6 & 0.7 \\
URM_H & 0.6 & 0.5 & 0.8 & 0.9 \\
RC_L & 0.6 & 0.5 & 0.8 & 0.9 \\
RC_M & 0.9 & 1.2 & 1.6 & 1.2 \\
RC_H & 0.9 & 1.2 & 2.1 & 1.2 \\
RCp_L & 0.9 & 0.9 & 1.3 & 1.2 \\
RCp_M & 0.9 & 1.2 & 2.1 & 1.2 \\
RCp_H & 0.9 & 1.2 & 2.1 & 1.2 \\
\hline
\end{tabular}

Table 8. $f_{\text {ag }}$ factors and macroseimic intensity increments $\Delta I$ evaluated according to Ambraseys et al. [18] predictive equation.

\begin{tabular}{lllll}
\hline \multirow{2}{*}{ Building Types } & \multicolumn{2}{c}{$\mathbf{f}_{\text {ag }}$} & \multicolumn{2}{c}{$\Delta \mathbf{I}$} \\
& Stiff & Soft & Stiff & Soft \\
\hline URM_L & 1.36 & 1.39 & 0.7 & 0.7 \\
URM_M & 1.33 & 1.45 & 0.6 & 0.8 \\
URM_H & 1.38 & 1.49 & 0.7 & 0.8 \\
RC_L & 1.38 & 1.49 & 0.7 & 0.8 \\
RC_M & 1.33 & 1.63 & 0.6 & 1.0 \\
RC_H & 1.33 & 1.68 & 0.6 & 1.1 \\
RCp_L & 1.40 & 1.59 & 1.0 & 1.0 \\
RCp_M & 1.34 & 1.65 & 1.1 & 1.1 \\
RCp_H & 1.29 & 1.57 & 1.0 & 1.0 \\
\hline
\end{tabular}

The implementation of the proposed period-depend approach for site effect amplification assessment within a seismic risk or a scenario analysis, require the identification of a common unit of analysis amongst the geotechnical zonation and the representation of the exposed building stock.

When exposure and seismic vulnerability are analysed for each single building, the information available on the building typology and class of height and the soil conditions underneath the analysed building can be used to evaluate the corresponding intensity increment $\Delta \mathrm{I}$ or, similarly, a soil amplification factor $\mathrm{f}_{\mathrm{ag}}$, according to Tables 6,7 or 8 .

When statistical data are available on small areas, e.g. census tracts, these have to be split into portions corresponding to the different soil categories therein identified. Centroids of these portions are therefore adopted as reference grid-points for the hazard evaluation and for the representation of the ground motion amplification due to soil conditions (either in terms of increments $\Delta \mathrm{I}$ for the macroseismic intensity or in terms of amplification factor $f_{a g}$ for response spectra). On the other hand, for the sake of a simplified operative approach, for each census track, reference can be made to the soil class more represent within the census tract.

The assessment of the macroseismic intensity increment $\Delta \mathrm{I}$ for as a function of both the soil conditions and the characteristics of the built environment, is made accounting for the ratio of building characterised by a the certain constructive material (unreinforced masonry, URM, pre-1970s reinforced concrete, $\mathrm{RC}_{\mathrm{p}}$, and reinforced concrete buildings, $\mathrm{RC}$ ) and a certain class of height:

$$
\Delta \mathrm{I}=\sum_{\mathrm{j}=1}^{3} \mathrm{RCp}_{\mathrm{j}} \Delta \mathrm{I}_{\mathrm{s}_{\mathrm{RC} p, \mathrm{j}}}+\sum_{\mathrm{j}=1}^{3} \mathrm{RC}_{\mathrm{j}} \Delta \mathrm{I}_{\mathrm{s}_{\mathrm{RC}, \mathrm{j}}}+\sum_{\mathrm{j}=1}^{3} \mathrm{URM}_{\mathrm{j}} \Delta \mathrm{I}_{\mathrm{s}_{\mathrm{URM}, \mathrm{j}}}
$$

where $R C p_{j}, \quad R_{j}, \quad U R M_{j}=$ proportion of pre-1970s reinforced concrete, reinforced concrete and unreinforced masonry buildings characterised by a specific class of height $(j=1$ low, $j=2$ medium, $j=3$ high);

$\Delta \mathrm{I}_{\mathrm{SRCpj}}, \quad \Delta \mathrm{I}_{\mathrm{RCj}}, \Delta \mathrm{I}_{\mathrm{SURMpj}},=$ macroseismic intensity increment attributed as a function of soil conditions $(\mathrm{S}=\mathrm{A}, \mathrm{B}, \mathrm{C}, \mathrm{D})$ and building types as in Tables 6,7 and 8 .

\section{EFFECTS OF ALTERNATIVE GEOTECHNICAL ZONATION APPROACHES ON THE ESTIMATION OF LOSSES}

A damage scenario analysis performed on a real study case is a very effective way to show the impact on the final results (expected consequences and uncertainties characterizing their estimation), of a geotechnical zonation performed according to different approaches and for different level of knowledge (information) available on the local site conditions. As a first step, these effects can be appreciated by comparing fragility curves or expected probabilities of exceeding pre-defined levels of damage, $\mathrm{D}_{\mathrm{k}}$, or potential consequences thresholds. Figure 2 shows, as an example, the effects of different soil conditions (represented in terms of EC8 soil classes) on the
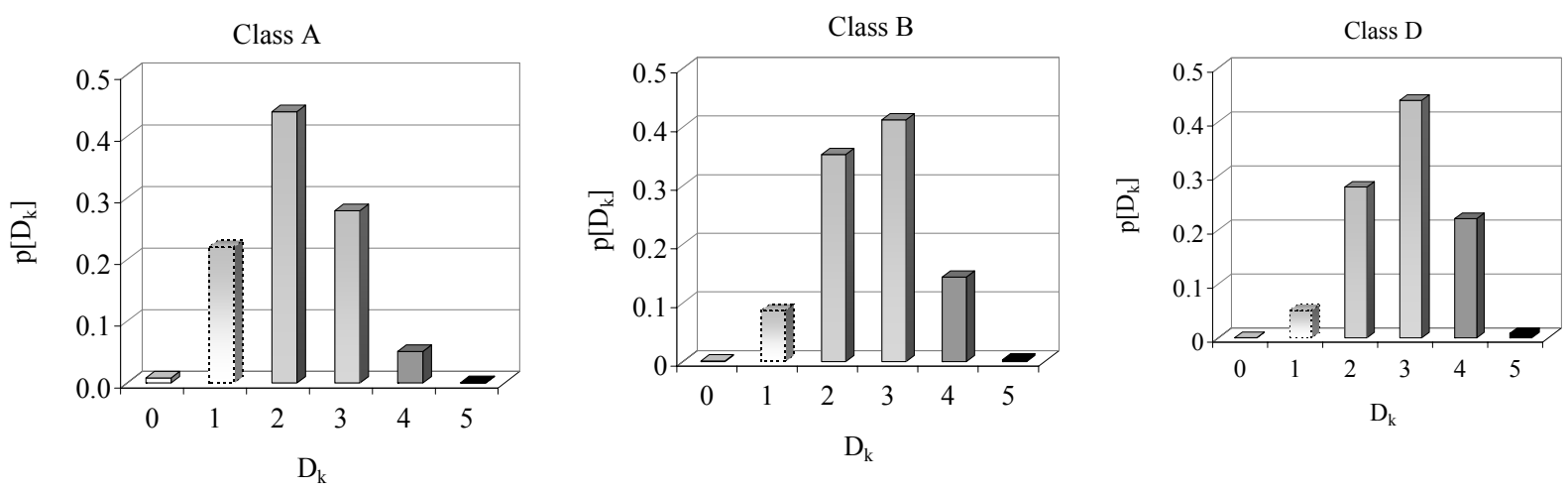

Figure 2. Damage probability distribution (EMS98 damage levels $D_{k} k=0 \div 5$ ) for unreinforced medium rise masonry buildings, URM_M, assuming an intensity $I_{E M S-98}=V I I I$ for different EC8 soil classes. 
expected levels of damage $D_{k}$ to existing buildings; the local ground motion amplification that have been considered are the ones derived from EC8 predefined spectral shape (according to what described before) assuming a value of the EMS-98 macroseimic intensity [19], $\mathrm{I}_{\mathrm{EMS}-98}=$ VIII evaluated on rock soil condition. The results are herein shown for the class of medium rise unreinforced masonry buildings, URM_M .

It is worth noting the substantial changes in the expected damage distribution, with higher probabilities of achieving the higher damage levels $D_{k}$ (e.g. moving from $D_{k}=2$ to $D_{k}=3$ ) and a substantial increase in the probability of reaching a heavy damage grade $p\left[D_{k}=4\right]$ beyond reparability level. Fragility curves can similarly provide a useful confirmation of these results. Figure 3 shows fragility curves related to the expected collapses, CB (Figure 3a) and uninhabitable buildings, UB (Figure $3 \mathrm{~b}$ ), for medium-rise masonry building types, built on different soil conditions.

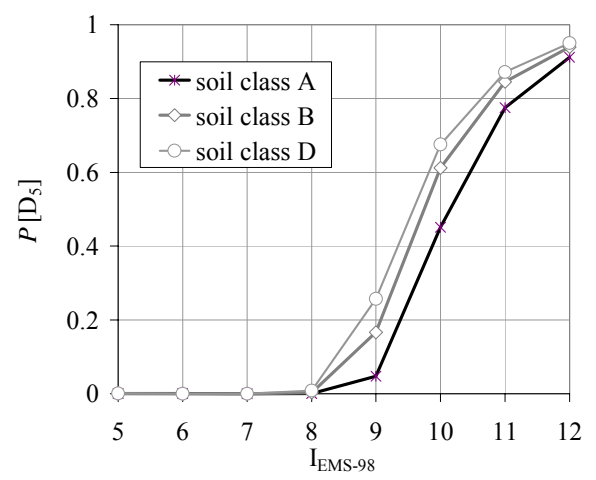

a)

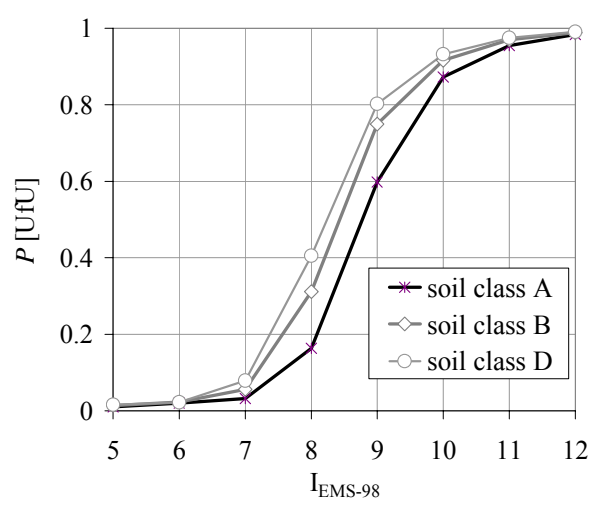

b)

Figure 3. Fragility curves for medium-rise masonry buildings on different soil classes: a) unfit for use buildings, b) collapsed buildings; obtained implementing the cross calibrated macroseismic-mechanic method, Giovinazzi and Lagomarsino [1]).
It is evident how a poor or improper definition of the geotechnical zonation and site conditions can play a major role within a seismic risk analysis at a territorial scale. Considering the impact and critical role of such analyses as a support to the decision making within urban or regional risk mitigation strategies (including seismic retrofit solutions), it can be argued that an appropriate reduction of the uncertainties related to the geotechnical hazard within a seismic risk framework can lead to more crucial and tangible benefits than in the case of the seismic response of a single building.

\section{EFFECTS OF ALTERNATIVE APPROACHES FOR THE DEFINITION OF SITE EFFECTS: A CASE STUDY}

It is evident that the different proposals available in literature as well as, within the same method, the different levels of refinement achievable to assess the geotechnical hazard can lead to substantially different representation of the zonation and of the amplification effects. As an example, a comparison of the results of the implementation of the two previously described, non-building dependant site effect amplification methods (TC4-ISSMGE, [2] and WP2-handbook by Faccioli and Pessina [6]) and of the building-depend approach for on the microzonation of a specific area, and on the assessment of the expected increment in the macroseismic intensity and therefore in the expected consequences, is carried out and herein discussed with reference to a case study. The region is represented by the Argentina Valley (Western Liguria, Italy), an area of about $30 \mathrm{Km}^{2}$, along the Argentina River, characterized by geological and topographic heterogeneity, as described in Isella et al. 2004 (Figure 4).

The region is characterised by an heterogeneous built environment, developed and concentrated in two main towns, Taggia and Arma and one small village, Castellaro.

The ancient town, Taggia, known as Tabia on the "Tavia fluvius" was an important Roman centre. The Lombard invasion in 641 forced its inhabitants to flee inland, where the mediaeval town, developed. Nowadays the built environment of the ancient town Taggia is still characterised by the total presence of unreinforced masonry building (Figure 6). The town of Arma, developed along a flat coastline, has been an important coastal area in the eighteenth and nineteenth century due to its shipyard and sea trading activities; the built environment of Taggia is characterised by reinforced concrete building mainly realised before 1971 (Figure 7). Castellaro is a small village built on a ridge. The name of this ancient town derives from "castellari", the strategic places that were given fortifications by local population. One of these was transformed into a castle, and the town grew up around it in the eleventh century; the built environment of the village is mainly characterised by medium rise unreinforced buildings. 


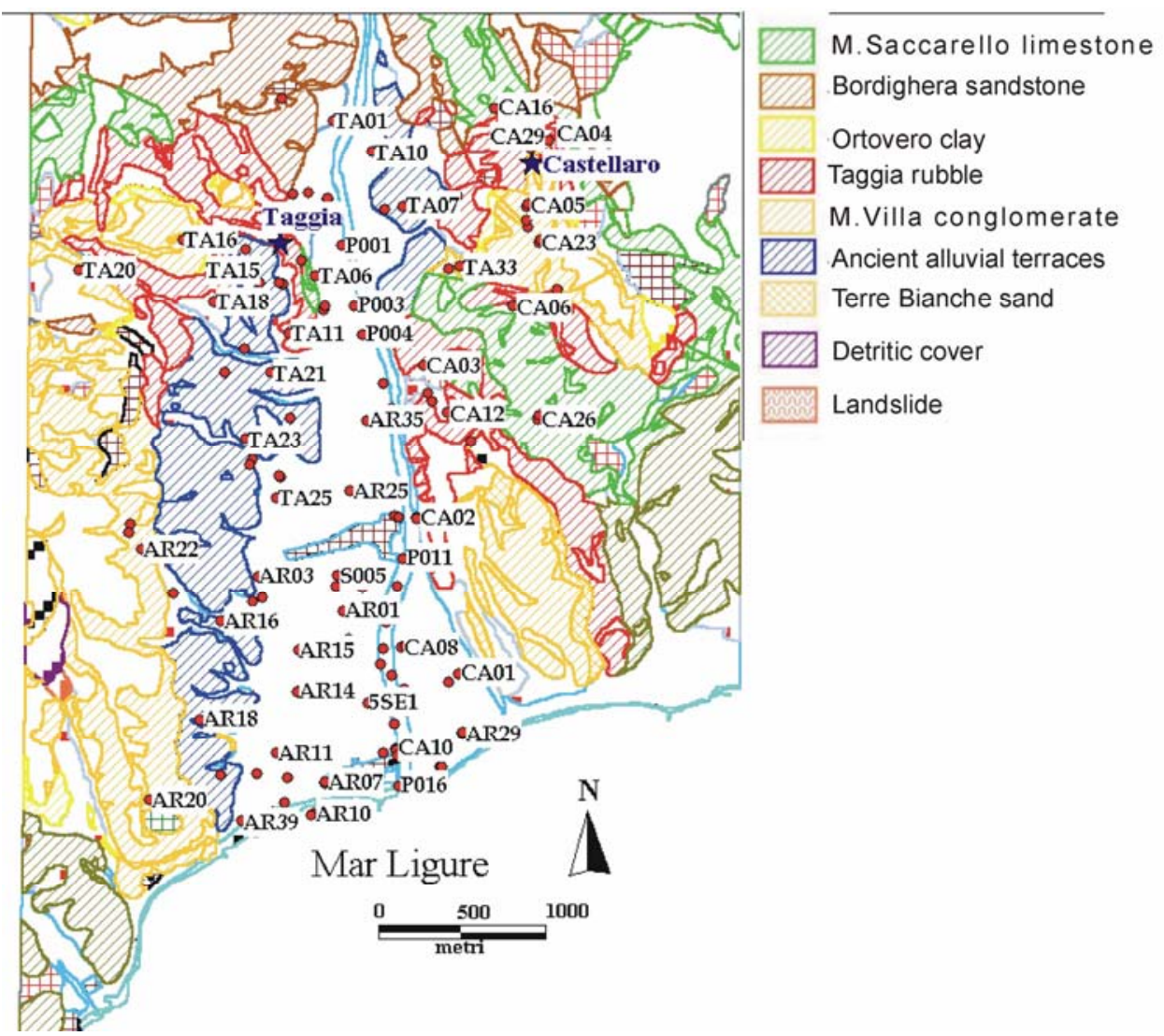

Figure 4: Geological-geomorphological map of the Argentina Valley and identification of noise measurements (red points) and velocimetric stations (blue stars).

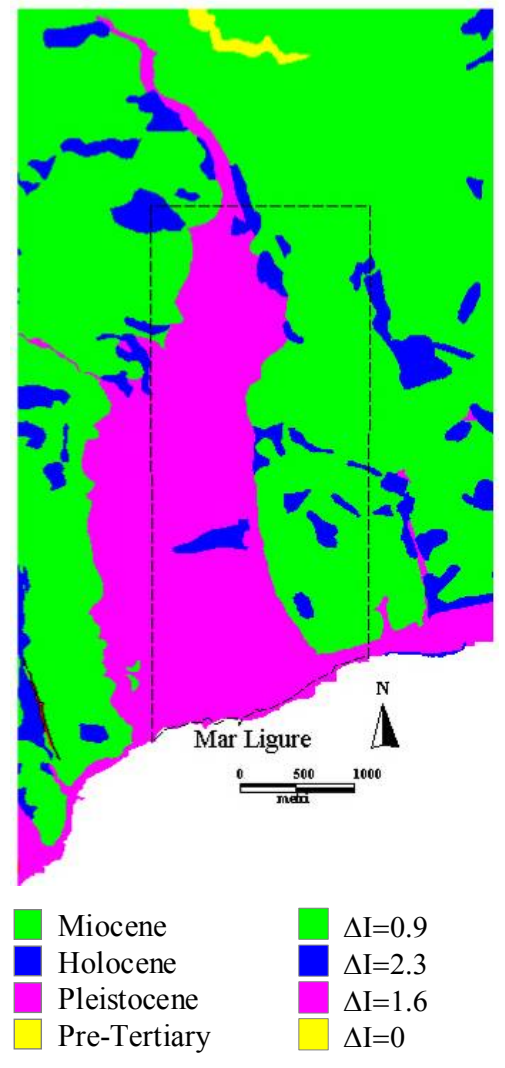

a)
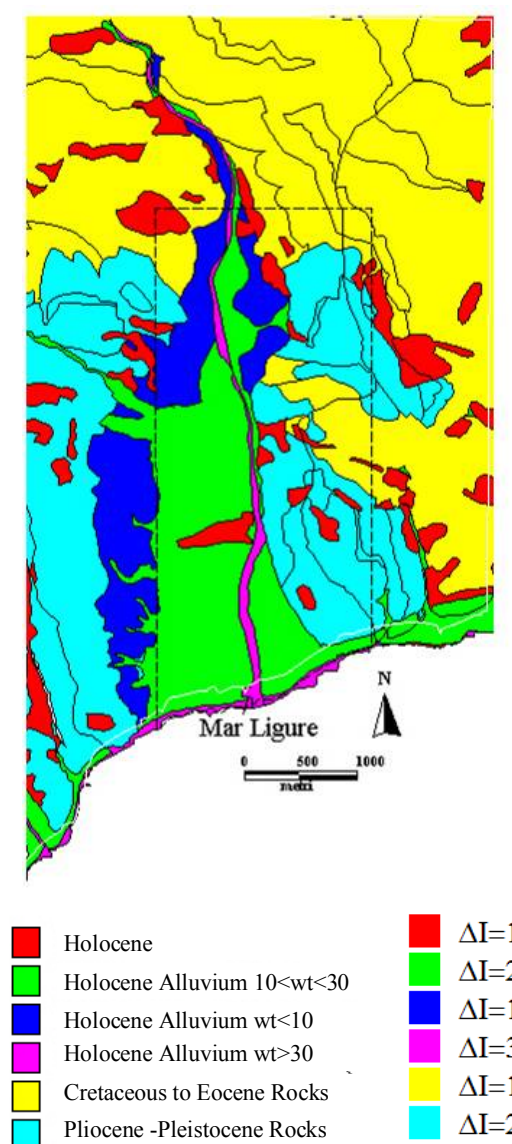

Plocene-Pleistocene Rocks

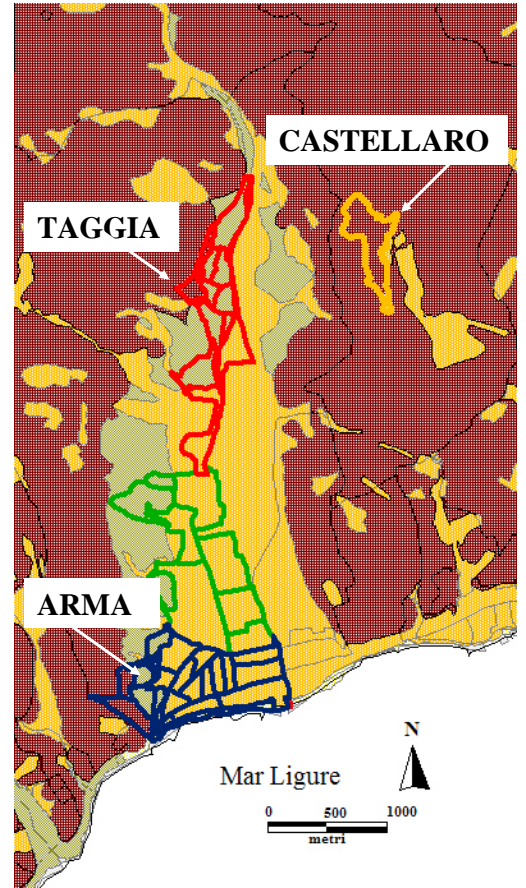

$\Delta \mathrm{I}=1.5$
$\Delta \mathrm{I}=2$
$\Delta \mathrm{I}=1.5$
$\Delta \mathrm{I}=3$
$\Delta \mathrm{I}=1.2$
$\Delta \mathrm{I}=2$

b)

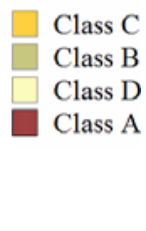

ass $\mathrm{C}$

lass B

Class D

$\Delta \mathrm{I}=0.5$

$\Delta \mathrm{I}=0$

$\Delta \mathrm{I}=1$

$\Delta \mathrm{I}=0$
$\Delta \mathrm{I}=1.2$ c)

Figure 5: Level-I zonation for the Argentina Valley according to: a) Midorikawa [5]; b) Everdnden and Thomson[4]; c) Faccioli and Pessina [6]. 
5.1 Level-I Zonation and assessment of non-period (nonbuilding) dependant site effect amplifications

Based on the available geological-geomorphologic map (Figure 4) a level/grade I zonation has been performed for the Argentina Valley according to the non-period dependant approaches for the assessment of local ground motion amplification due to site conditions, described in Par. 2.1.

Figures $5 \mathrm{a}$ and $5 \mathrm{~b}$ show a TC4 grade-I zonation, performed making reference to the available geological map in Figure 4, according to the recommendations provided by Midorikawa [5] (Figure 5a) and by Everdnden and Thomson [4] (Figure $5 \mathrm{~b}$ ). Figure $5 \mathrm{c}$ shows a level-I zonation performed according to the handbook for earthquake ground motion scenarios of the Risk-UE project [6]. The near-surface formations of the available geological map (Figure 4) have been interpreted in terms of approximate geotechnical units corresponding to EC8 [9] ground classes.

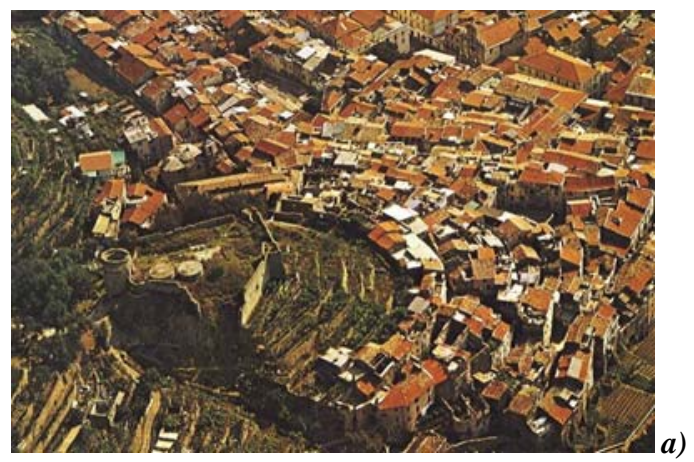

Comparing the maps represented in Figure 5a, 5b, 5c, significant differences in terms of the resulting microzoning and in the attribution of the expected macroseimic intensity increments $\Delta \mathrm{I}$ can be observed. The differences observed in the attribution of the macroseimic intensity have been summarised in Table 9, for the areas corresponding to the three towns under analysis (Arma, Taggia and Castellaro). Regarding the resulting microzoning, it is worth highlighting that Midorikawa [5] and Everdnden and Thomson [4] approaches do not allow for a detailed microzonation. When implementing the two methods a uniform litology is recognised for each one of three cities (Figure 5a and Figure $5 \mathrm{~b}$ ), and consequently a same intensity increment is attributed for each entire city (Table 9). This leads to an inaccurate and rough representation of the site response that, as known, can vary significantly for regions only a few kilometres apart [20]. On the other hand, when performing the microzonation according to Faccioli and Pessina approach [6], differences in the identification of the soil classes can be appreciated at the level of each single census tract within the city (Figure $5 \mathrm{c}$ ).

Figure 6: Taggia town: a) picture of the historical centre showing the tight aggregation of the masonry built-
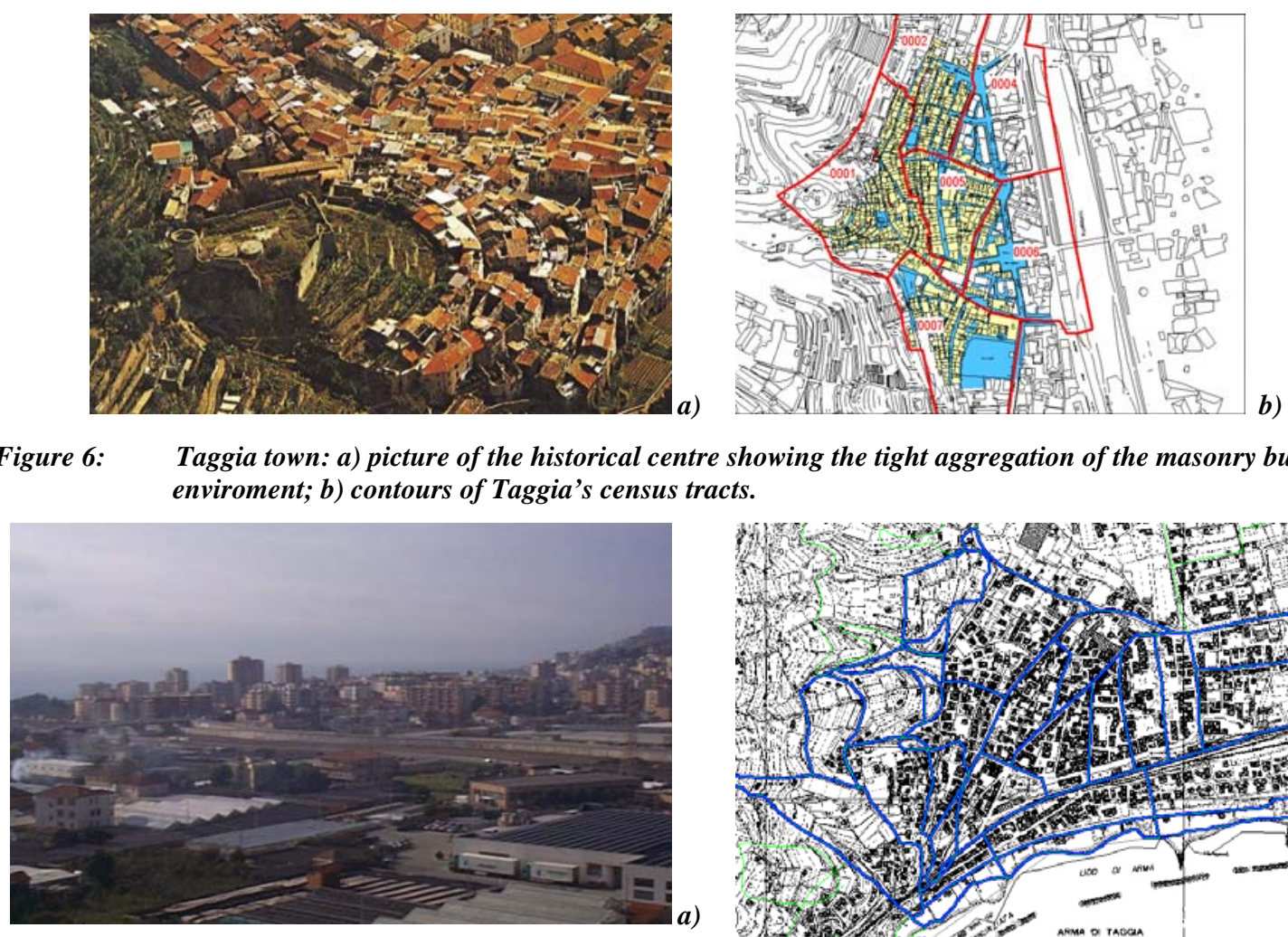

\section{enviroment; b) contours of Taggia's census tracts.}

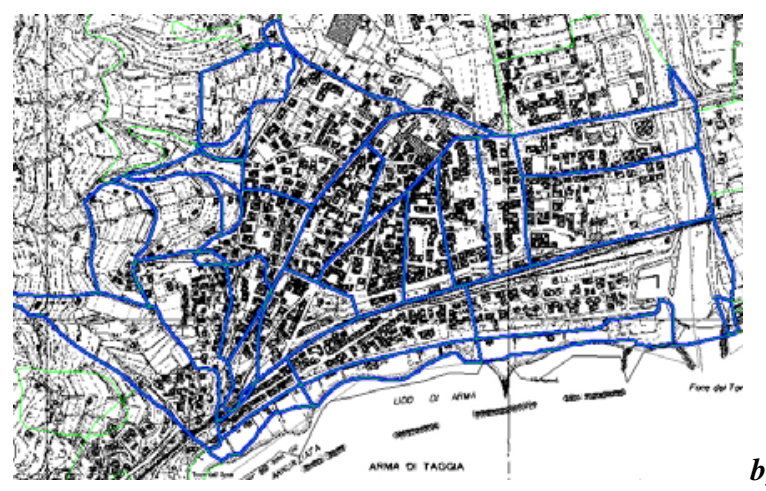

Figure 7: Arma town: a) picture of the coastline of Arma showing the prevailing high-rise reinforced concrete building typologies; b) contours of Arma's census tracts.
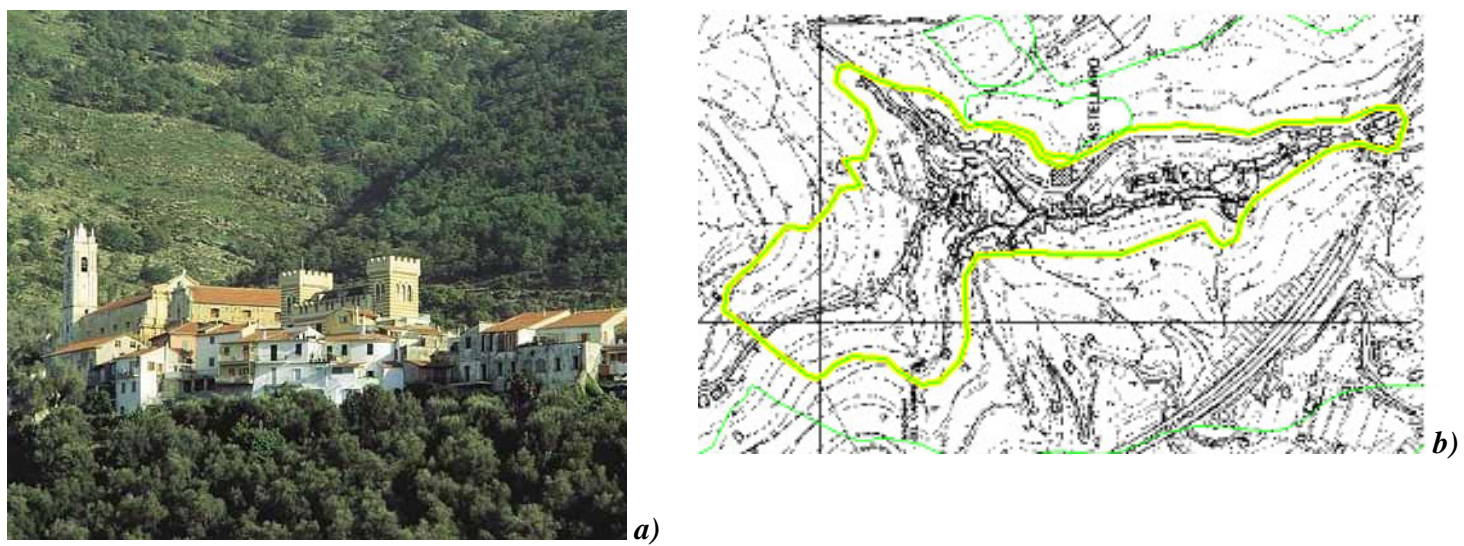

Figure 8:

Castellaro village: a) built environment and site morphology ; c) contours of Castellaro's census tract. 
Depending on the prevalent soil class recognised within the census tract and according to the values presented in Table 4, a different intensity increment has been attributed to each census tract (Table 9). Regarding the intensity increments it is worth noting that both for the towns of Arma and Taggia, the macroseismic increments attributed according to Midorikawa [5] and Everdnden and Thomson [4] approaches overestimate, from two to three times, the ones recognised according to Faccioli and Pessina approach [6]. Regarding to Castellaro village, while no intensity increment due to soil effect has been recognised according to Faccioli and Pessina [6] approach, a macroseismic intensity increment in the range $\Delta \mathrm{I}=$ 1.5-0.9 has been recognized according to the other approaches.

Table 9. Macroseimic intensity increments $\Delta I$ evaluated, for the three towns under analysis according to the nonperiod dependent zonation approaches.

\begin{tabular}{lccc}
\hline & E.\&T. (1985) & M. (1987) & F.\&P. (2003) \\
& $\Delta \mathbf{I}_{\text {MM }}$ & $\Delta \mathbf{I}_{\text {MM }}$ & $\Delta \mathbf{I}_{\text {EMS98 }}$ \\
\hline Arma & 2 & 1.6 & 0.5 or 1 \\
Taggia & 1.5 & 1.6 & 0 or 0.5 \\
Castellaro & 2 & 0.9 & 0 \\
\hline
\end{tabular}

\subsection{Level -II Zonation}

A level-II zonation, according to the Risk-UE guidelines [6], has been performed (Figure 9) by using available geophysical profiles and seismic measurements [21]. In particular, the seismic data set consisted of weak motion recordings, collected by a local temporary network, and of micro-tremor data recorded at 150 noise measurements points (Figure 4). The method proposed by Nakamura [22], has been implemented for the evaluation of the site response from the acquired microtremors, in terms of the Fourier spectral ratio of horizontal versus vertical component (H/V spectral ratio). The reliability of the site response estimation so obtained has been cross-validated with the ratio of the horizontal spectra from the weak motion recordings. Moreover, a good agreement has been observed comparing the $\mathrm{H} / \mathrm{V}$ spectral ratios with the transfer functions obtained from one-dimensional numerical simulations. From the frequencies of the dominant peak in the spectral ratios of horizontal to vertical motion evaluated on the irregular grid of observation points, a continuous map of the fundamental resonance frequency (Figure 9a) was derived by interpolation using the features of the Geographical Information System (GIS) software Mapinfo ${ }^{\circledR}$. The use of the GIS software allowed, furthermore, for the construction of a subsurface model by integrating geological and geophysical profiles (Figure 9b), available from a previous study (Peloso personal communication). The subsurface model led to the definition of a map of the soil thickness. The average shear wave velocity in the uppermost $30 \mathrm{~m} \mathrm{Vs}_{30}[\mathrm{~m} / \mathrm{s}]$ map (Figure 9c) was obtained, for the studied region, combining the information provided by the frequency and the thickness maps.

As a function of the values obtained for the parameter $\mathrm{Vs}_{30}$ and making reference to soil classification scheme adopted within the Eurocode EC8 (Table 4), the map of the soil classes in the region has been drawn, provided confirmation of the results obtained implementing the Risk-Ue Level I approach (Figure 5c).

For the same area and relying on the same set of available data, a level-II microzoning has been performed in terms of Resonance frequencies [23], confirming the soil classification obtained in terms of $\mathrm{Vs}_{30}$.

\subsection{Assessment of period-dependant site effect amplifications}

The assessment of the macroseismic intensity increment $\Delta \mathrm{I}$ as a function of both the soil conditions and the characteristics of the built environment, has been performed for the Argentina Valley according to the period-dependant approach presented in Par. 3.2.

The ratio of buildings characterised by a the certain constructive material (unreinforced masonry, URM, pre-70
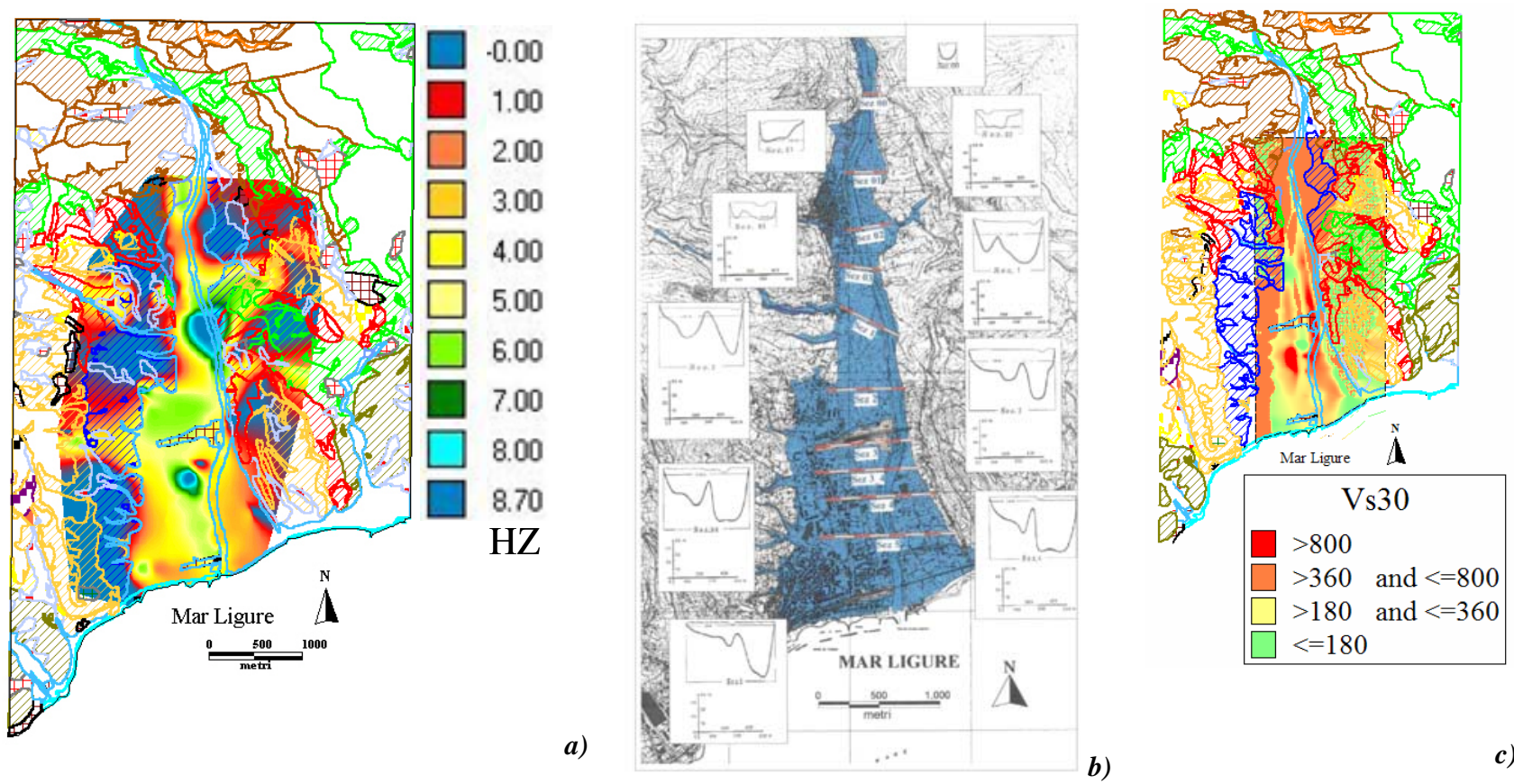

Figure 9: II-level zonation for the Argentina Valley: a) map of the fundamental frequencies (Hz) from noise measurements; b) map of S.E.V (vertical geoelectrical boreholes); c) map of the shear wave velocity in the uppermost $30 \mathrm{~m} V s 30[\mathrm{~m} / \mathrm{s}]$. 
reinforced concrete, $\mathrm{RCp}$, reinforced concrete, $\mathrm{RC}$ buildings) and a certain class of height, (according to the classification scheme adopted in Tables 6,7,8), has been assessed making reference to the statistical data about the built-environment available for all the Italian territory [24].

For each census tract, a representative soil class has been recognised making reference to the microzonation performed in term of Eurocode EC8 soil classes $(\mathrm{S}=\mathrm{A}, \mathrm{B}, \mathrm{C}, \mathrm{D})$ presented in Figure 5a.

Maps in Figures 10a and 10b show the distribution in term of medium high masonry typologies, URM_M, and pre-70 reinforced concrete typologies, low-rise, RCp_L, medium-rise, $\mathrm{RCp}$ M, high-rise, RCp_H respectively for Taggia and Arma towns. Unreinforced masonry buildings are prevalent in Taggia, concentrated in the area of the historical centre, while in the adjacent areas low-rise and medium-rise reinforced concrete building co-exist with the unreinforced masonry ones (Figure 10a). Conversely, Arma is mainly characterized by reinforced concrete buildings, principally high-rise in the costal areas and medium-rise in the inner areas (Figure 10b). As per the soil classes, Taggia is mainly located on a soil recognized as Class $\mathrm{B}$, with three census tracts located in Class C and one census tract in Class A (Figure 5a). Arma is largely located on a soil of Class $\mathrm{C}$, expect for the shore line, classified as Class D and few census tracts, scarcely populated, recognized as Class A/B (Figure 5a).

For each census tract, as a function of both the ratio of unreinforced masonry and reinforced concrete building belonging of a specific class of height, and of the prevalent soil class, the macroseimic intensity increment $\Delta \mathrm{I}$, has been estimated according to Equation 5. No macroseismic intensity increments results for Taggia historical centre, $\Delta \mathrm{I}=0$, while for the adjacent areas the expected macroseimic intensity increments is in the range of $\Delta \mathrm{I}=0.25 \div 1$ (Figure 11a).

As per Arma town, an intensity increment in the range of $\Delta \mathrm{I}=$ $1.25 \div 2$ results for the costal area; in the inner areas, depending on the characteristic of the built environment, a macroseismic intensity increment in the range $\Delta \mathrm{I}=1 \div 1.25$ has been assessed for few census tracts, being $\Delta \mathrm{I}=0.75 \div 1$ for the others (Figure 11b). The macroseismic intensity increment results $\Delta \mathrm{I}=$ $0.75 \div 1$ for the census tracts characterised by soil classes $A$ and B (Figure $11 \mathrm{~b}$ and Figure 5c).

Comparing the results obtained implementing the proposed period-depend approach with the ones assessed according to the non-period dependent ones (Tab. 9), it is worth highlighting, how: 1) the period-dependant approach allow to differentiate, for a same class of soil, the expected amplification of the macroseismic intensity depending on the characteristic of the built-environment; 2) the results from the period dependent approach are included in the superior bounds provided by Everdnden and Thomson [4] and Midorikawa [5] approaches and the inferior bound resulting from the implementation of the approach proposed by Faccioli and Pessina [6].

\subsection{Effects of alternative assessment of the local ground motion amplification due to soil conditions}

In order to asses the effects of alternative assessment of the local ground motion amplification due to soil conditions on the estimation of the expected damage and consequences, a damage scenario analysis has been performed for the studycase. To this aim, the maximum historical event in the region has been considered, corresponding to the Western Liguria Feb 23, 1887 earthquake $\left(\mathrm{M}=6.3, \mathrm{I} 0=\mathrm{X}\right.$, Long $=8^{\circ}, 1430$, Lat $=43^{\circ}, 7480$ ), which caused over 509 victims, severe destruction in costal towns and villages. The vulnerability assessment has been performed according to the macroseismic method [1], taking into account the data about the typological and constructive features of the built-environment, provided by census data [24]. The expected structural and non structural damage to the building, has been estimated in terms of the EMS-98 damage scale (five damage grades $D_{k} k=0 \div 5$ : slight damage, $D_{1}$, moderate damage $D_{2}$, heavy damage $D_{3}$, very heavy damage $\mathrm{D}_{4}$, destruction $\mathrm{D}_{5}$, plus the absence of damage $\mathrm{D}_{0}$, no damage).

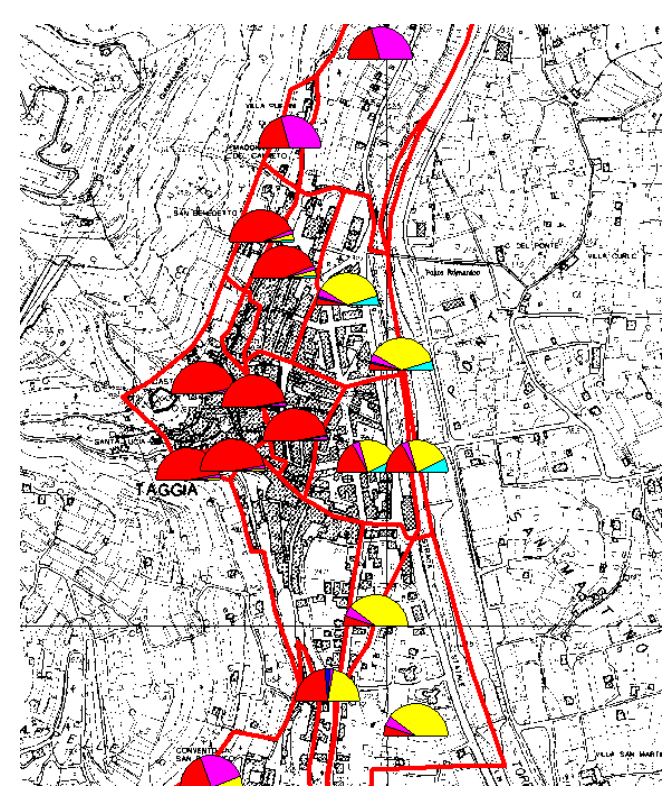

a)

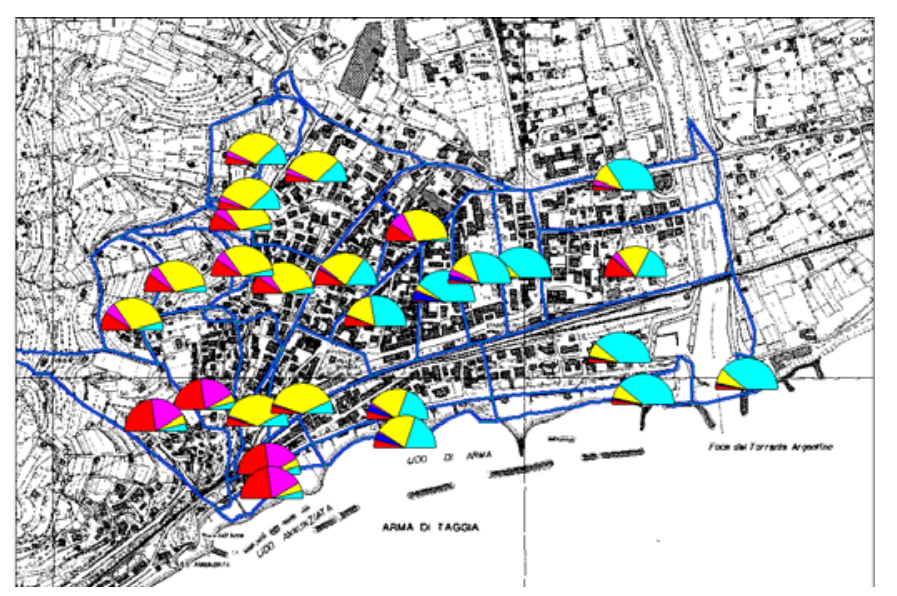

b)

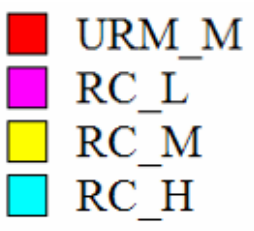

Figure 10: Building typology distribution for: a) Taggia town; b) Arma town. 


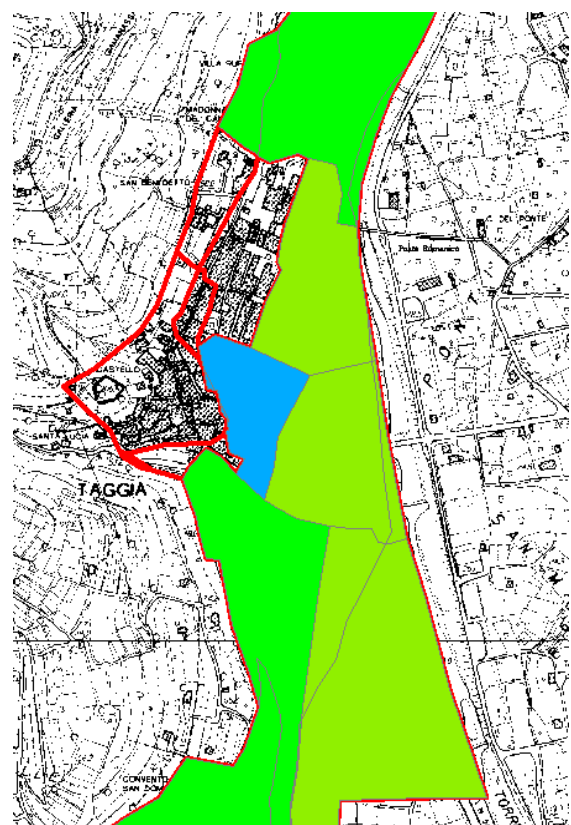

a)

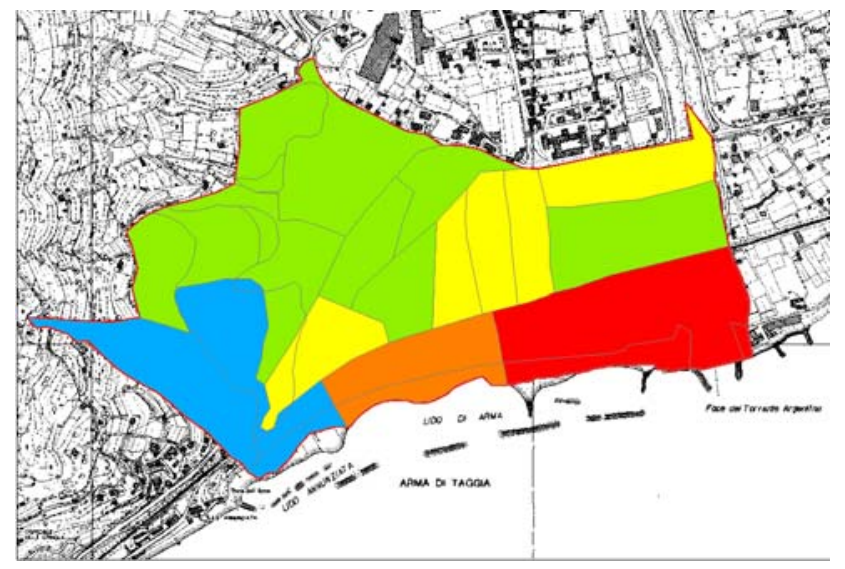

b)

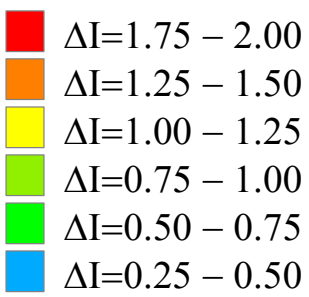

Figure 11: Macroseismic Intensity Increments $\Delta I$ evaluated according to the period dependant approach for: a) Taggia town; b)Arma town.

For Arma town, the expected damage distributions has been evaluated for the different hypothesis of macroseismic intensity increments, assessed according to two non-period dependant approaches, namely Everdnden and Thomson [4], and Faccioli and Pessina [6], and according to the proposed period dependant-approach [1]. The resulting damage distributions are represented, respectively, in Figures 12a, 12b, and $12 \mathrm{c}$.

It is worth noting the substantial changes in the expected damage distribution with: 1) high probabilities of achieving a damage level $\mathrm{D}_{2}$ after the assessment performed according to Faccioli and Pessina [6], represented in Figure 12b; 2) higher probabilities of achieving a damage level $\mathrm{D}_{3}$ in the implementation performed according to Giovinazzi and Lagomarsino [1], Figure 12c;3) a substantial increase in the probability of reaching a heavy damage grade, $\mathrm{D}_{4}$, when the expected macroseismic increments are evaluated according to approach proposed by Everdnden and Thomson [4], in Figure $12 \mathrm{a}$.

The impact of the different assessments can be furthermore appreciated by comparing the estimation of the consequences expected on people and building.

The assessment of the consequences on building and people has been performed, as a function of the structural and non structural damage expected for the buildings, according to empirical correlations based on observed data [25]. The diagrams in Figure 13a and 13b show the percentage of collapses, CB and uninhabitable buildings, UB, homeless people, HP, casualties, $\mathrm{C}$ expected for two census tracts of Taggia town: 1 ) census tract $\mathrm{N}^{\circ} 18$ (number of building $=26$; number of resident people $=201)$; 2) census tract $\mathrm{N}^{\circ} 27$ (number of buildings $=50$; number of resident people $=418$ ). In Figure 12a census tracts 18 and 27 are identified, and from Figure 10b, where the building typology distribution is represented for Arma town, it can be seen how high-rise and medium-rise reinforced concrete buildings are prevalent respectively in sections 18 and 27. When implementing Everdnden and Thomson approach [4], the same litology is recognised, namely "Holocene Alluvium", for both the census tracts and the same intensity increment is attributed $\Delta \mathrm{I}=2$ to the two census tracts (Figure 5b). Because of that the differences in the damage distribution (Figure 12a) and in the consequences estimation (Figure 13), when implementing Everdnden and Thomson approach are due only to the differences in the building vulnerability assessment. On the other hand, when implementing Faccioli and Pessina approach [6], a different soil class is recognised for the two census tracts, namely soil $\mathrm{C}$ for census tract 18 , and soil B for census tract 27 (Figure 5c). This lead to a different attribution of the intensity increments corresponding to $\Delta \mathrm{I}=0.5$ for census tract 18 and $\Delta \mathrm{I}=0$ for census tract 27 (Figure $5 \mathrm{c}$ ), and a consequent different estimation of the damage distribution (Figure 12b) and expected consequences (Figure 13). Finally, the implementation of Giovinazzi and Lagomarsino approach [1], allow to account for the peculiar situation, on one hand, of census tract 18, where the prevalence of high-rise reinforced concrete structures are built on a soft soil, class C, and on the other hand, of census tract 27, where the prevalence of medium-rise reinforced concrete structures are built on soil, class $B$. This lead to a different attribution of the macroseismic intensities for the two census tracts, corresponding to $\Delta \mathrm{I}=1.8$ and $\Delta \mathrm{I}=0.8$ respectively for census tract 18 and 27 (Figure $11 \mathrm{~b})$ and to a different assessment of the resulting damage distribution (Figure 12c) and expected consequences (Figure 13). It is worth highlighting that the expected consequences, evaluated according to the period-dependant approach, are always comprised between the ones assessed with the two non-period dependant approaches. 

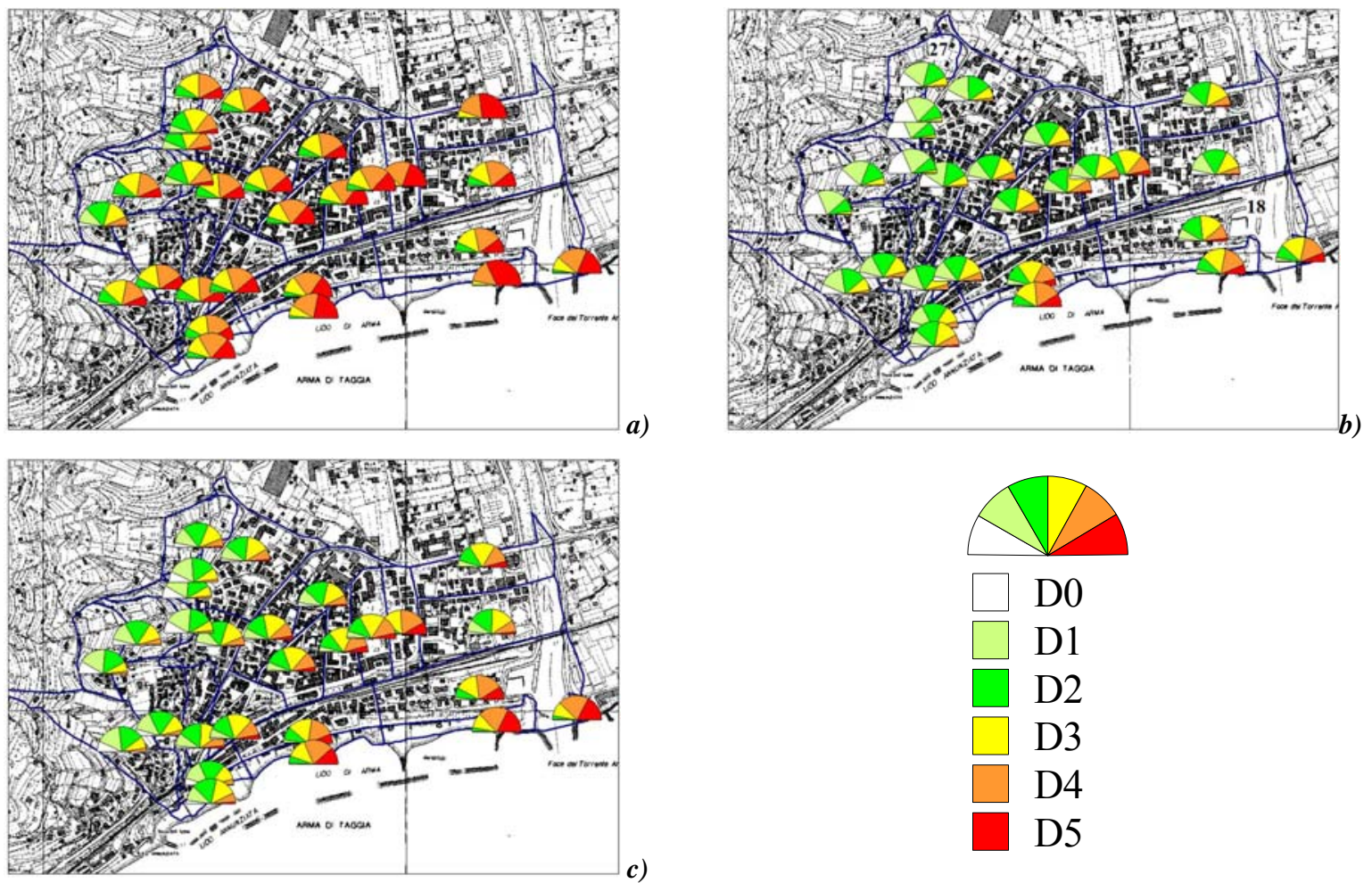

Figure 12: $\quad$ Expected damage distribution for Arma town, for the 1887 scenario, evaluated accounting for the site effect macroseismic intensity increment, $\Delta I=1$ according to: a) Midorikawa [5]; b) Faccioli and Pessina [6]; c) Giovinazzi and Lagomarsino [1].
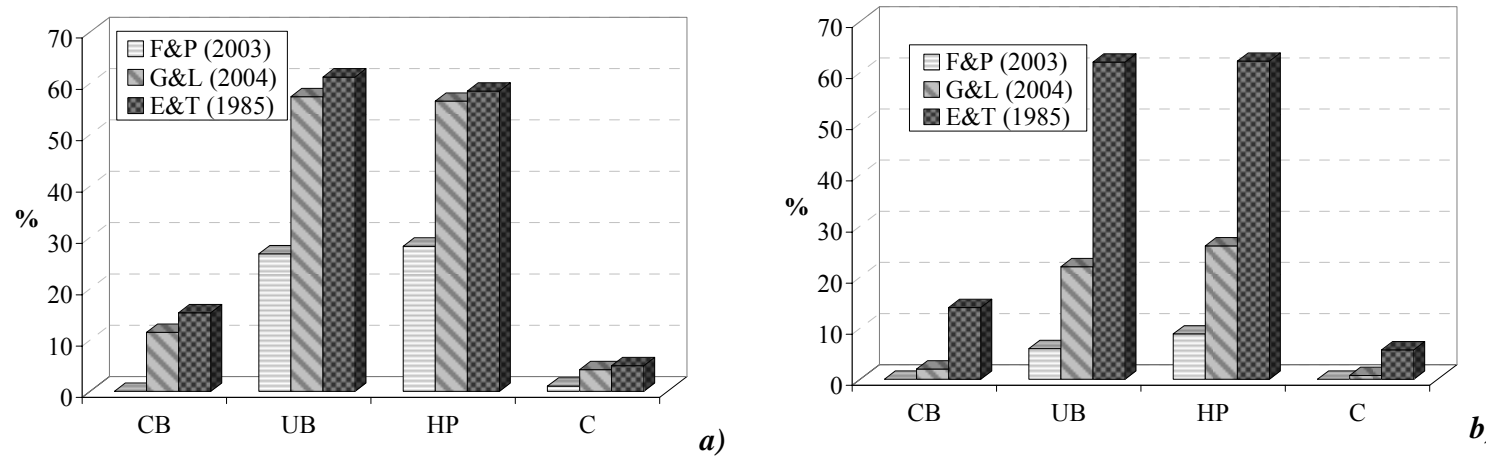

Figure 13: Expected consequences for Arma, for the 1887 scenario, evaluated for different site effects assessments, census tract: a)18; b)27. 


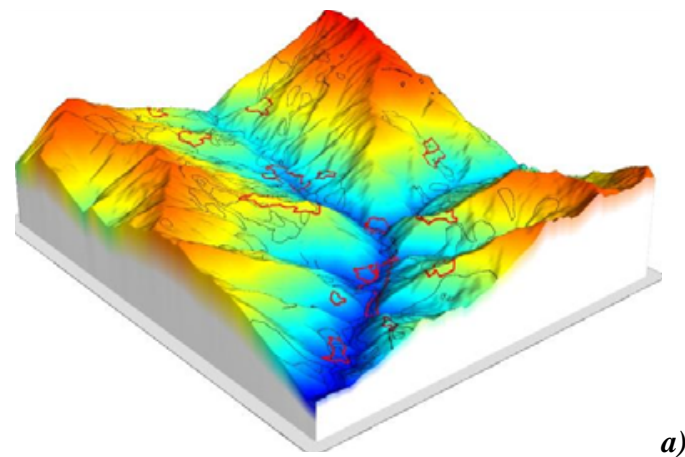

a)

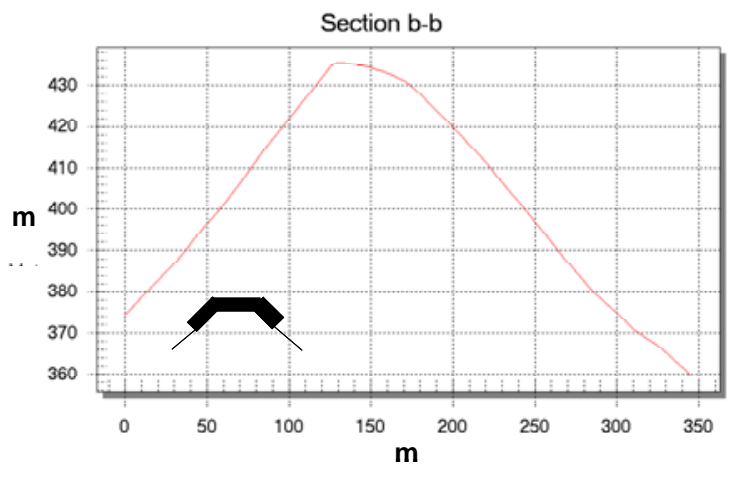

c)
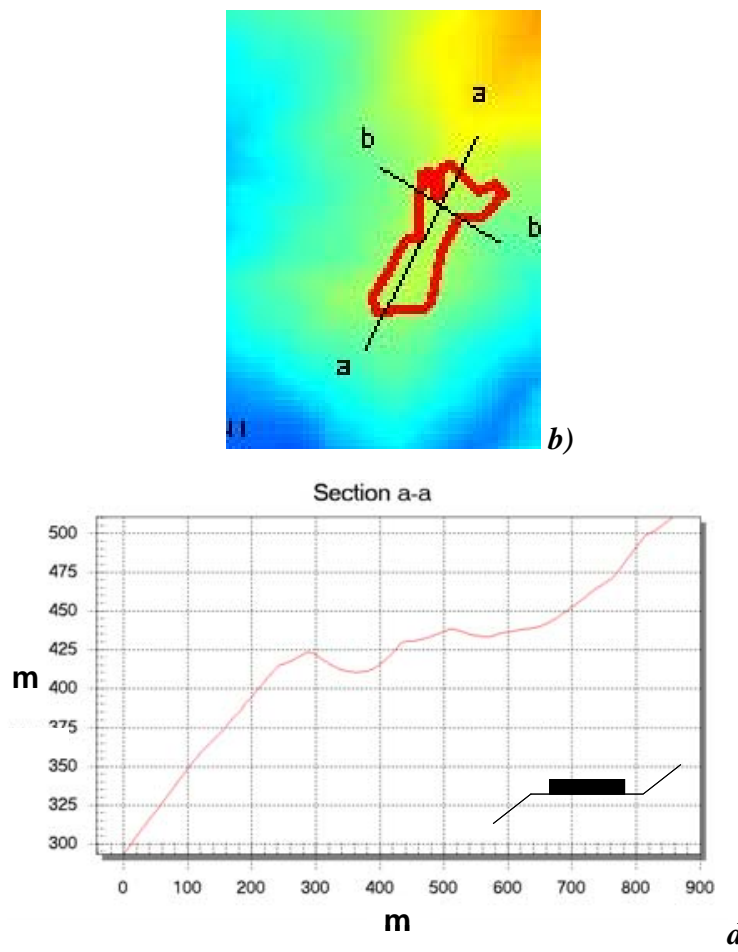

d)

Figure 14: Identification and classification of the irregular surface profile for Castellaro village: a) Three-dimensional representation of the DEM; b) identification of two perpendicular sections; $c$ ) d) slope profiles corresponding to the two sections and qualitative simplified representation.

The investigation of the potential local ground motion amplification due to surface irregularities, according to the simplified assessment presented in Par. 2.2, was performed for Castellaro village. Castellaro is located on a rock ridge crest that could potentially cause amplification due to surface irregularities, as already observed after historical earthquakes [11].

In order to identify the shape of the ridge where Castellaro is located and to classify that according to the irregular surface profile classification adopted in the literature (Tab. 5), the variability in slope and aspect of the surface morphology has been observed by the use of a Digital Elevation Model, DEM.

A DEM is a raster or grid based terrain model. Each cell in the DEM is characterised by three coordinates, where the $\mathrm{Z}$ coordinate is representing the elevation of the area. A digital elevation model, DEM, can be defined as a numerical model of a terrain surface, which along with a mathematical method of interpolation makes it possible to calculate the (surface) elevation of any point within the domain of the model.

The MapInfo GIS ${ }^{\circledR}$ extended with the Vertical Mapper ${ }^{\circledR}$ module, has been used to implement the shape profile of Castellaro ridge from the DEM data provided by Liguria Region. First of all, a three-dimensional DEM of the area has been drawn and contours of the census tracts corresponding to the different villages and town located in the area have been overlaid (Figure 14a). Secondly, the slope profiles (Figure 14b and Figure 14c) corresponding to two perpendicular sections identified for Castellaro village (Figure 14b), have been represented. Finally, a qualitative spatial patterns recognition has been performed in order to identify the morphologic profile according to the simplified classification proposed in Tab. 5.

Implementing the aforementioned procedure, Castellaro has been recognised as a ridge with a crest and width minor of the base, and with an average slope angle inferior to $30^{\circ}$. Therefore an intensity increment $\Delta \mathrm{I}=1$, has been hypostatised for Castellaro village, according to what suggested in Table 5 .
The histograms in Figure 15 show the differences in the expected percentages of Collapsed Buildings, CB, Uninhabitable Buildings, UB, Homeless People, HP, casualties, $\mathrm{C}$ expected for Castellaro (total number of Buildings $=230$; People $=441$ ), for the 1887 earthquake scenario, evaluated on one hand accounting for the Intensity increment, $\Delta \mathrm{I}=1$ due to surface irregularities, and, on the other hand, neglecting the potential intensity increment, $\Delta \mathrm{I}=0$.

It is worth highlighting how the expected consequences would result from three to four times higher when accounting for a potential amplification of the ground motion due to surface irregularities.

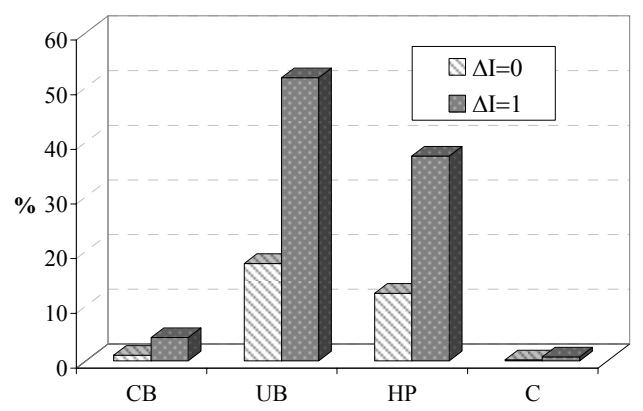

Figure 15: Expected consequences for Castellaro, for the 1887 scenario, when accounting for the intensity increment, $\Delta I=1$ due to surface irregularities, and when neglecting that, $\Delta I=0$.

\section{CONCLUSIONS}

An appropriate representation of the geotechnical hazard, able to account for both the local ground motion amplification due to soil and morphological conditions as well as the induced potential hazards (e.g. liquefaction ground settlements and landslides phenomena) is a critical step of refined seismic risk scenario study. Lack of appropriate information as well as scarce zoning characterization can lead to substantially unconservative results in terms of assessment of the seismic risk, 
thus impairing the implementation of cost-efficient risk mitigation strategies.

In this contribution, focus has been given on the discussion of alternative methods for the evaluation, at territorial scale, of site effects due to soil and morphological conditions. Alternative methods able to account for both the soil conditions and the characteristics of the built environment have been also discussed, proposing different formulations depending on the vulnerability methods adopted. In particular, when a macroseismic vulnerability approach is used, macroseismic intensity increments $\Delta \mathrm{I}$ can be evaluated for different building typologies and soil classes, referring to either seismic code response spectra or to attenuation laws available in literature.

An example of the effects of implementing different geotechnical zonation methods has been provided with reference to a case-study, identified with the Argentina Valley (Western Liguria, Italy). Significant differences have been observed in terms of either the microzoning maps as well as of the quantitative representation of the amplification effects.

The influence of the geotechnical zonation on the results of a risk analysis have been shown in terms of the variation of the expected distribution of damage and casualties. To this aim, a damage scenario analysis for the case-study has been presented, in order to compare and discuss the difference on the results depending on the approach adopted for the seismic zonation and for the representation of local morphological and site effects amplification.

\section{REFERENCES}

1 Giovinazzi, S. and Lagomarsino, S. (2004). "A Macroseismic Model for the vulnerability assessment of buildings". Proc. 13th World Conference on Earthquake Engineering, Vancouver, Canada, paper 896.

2 TC4-ISSMGE (1993; revised 1999) Manual for Zonation on seismic Geotechnical Hazards. Technical Committee for Earthquake Geotechnical Engineering.

3 Medvedev, J. (1962). Engineering Seismology. Academia Nauk Press, Moscow.

4 Everdnden, J., Thomson, J.M. (1985). "Predicting Seismic Intensities". U.S. Geol. Survey. Paper 1360.

5 Midorikawa, S. (1987). "Prediction of Isoseismal map in the Kanto Plain due to hypothetical Earthquake". Journal of Structural Engineering. 33B. (in Japanese).

6 Faccioli, E. and Pessina, V. (2003). "WP2-Basis of an handbook of earthquake ground motion scenarios". RiskUE Project An advanced approach to earthquake risk scenarios with applications.

7 RISK-EU (2004). "The European Risk-Ue Project: An Advanced Approach to Earthquake Risk Scenarios". (2001-2004) www.risk-ue.net.

8 Bard, (1998). "SERINA: Seismic Risk and Integrated Seismological, Geotechnical and Structural Approaches". ITSAK, European Commission, Directorate General for Science and Development.

9 CEN (2004). Eurocode 8: Design of structures for earthquake resistance - Part 1: General rules, seismic actions and rules for buildings. European Committee For Standardisation (CEN), Brussels.

10 Paolucci, R. and Rimordi, A., (2002). "Seismic amplification for 3D steep topography irregularities". Proc. of 12th European Conference on Earthquake Engineering, London.
11 Faccioli, E., Vanini, M., Frassine, L. (2002). "Complex" site effects in earthquake round motion, including topography. Proc. of 12th European Conference on Earthquake Engineering, London.

12 Siyahi, B.G. and Ansal, A. (1993) in TC4-ISSMGE (1993; revised 1999) Manual for Zonation on seismic Geotechnical Hazards. Technical Committee for Earthquake Geotechnical Engineering, 68-70.

13 Bird, J.F., Bommer, J.J, Crowley, H., Pinho, R. (2006). "Modelling liquefaction -induced building damage in earthquake loss estimation". Soil Dynamics and Earthquake Engineering. 26(1). 15-30.

14 Giovinazzi, S. and Cubrinovski, M. (2007). "Liquefaction Hazards for Seismic Risk Analysis". Proc. of 2007 NZSEE, Conference of the New Zealand National Society for Earthquake Engineering.

15 NIBS (1999). HAZUS Earthquake Loss Estimation Methodology: User's Manual, Federal Emergency Management Agency (FEMA), National Institute of Building Sciences, Washington, DC. 1, 2, 3.

16 Giovinazzi, S. (2005). "The vulnerability assessment and the damage scenario in seismic risk analysis". Ph.D Thesis of the doctoral course "Risk Management on the built environment" jointly organized by University of Florence (I) and TU-Braunschweig (D).

17 Chopra, A.K. and Goel, R.K. (1999). "CapacityDemand-Diagram methods based on inelastic spectrum". Earthquake Spectra. 15(4). 637-656.

18 Ambraseys, N.N., Simpson, K.A., Bommer, J.J. (1996). "Prediction of horizontal response spectra in Europe". Earthquake Engineering and Structural Dynamics. 25. 371-400.

19 Grunthal, G. (1998). European Macroseismic Scale. Centre Européen de Géodynamique et de Séismologie, Luxembourg. Vol. 15.

20 Boore, D.M. (2004). "Can site response be predicted". J. Earthquake Engineering. 8. no. S1, 1-41.

21 Isella, L., Giovinazzi, S., Spallarossa, D., Eva, C., (2004). "Site-Specific Microzonation Study in Northwestern Liguria". 29th General Assembly of ESC, Potsdam, Germany. (Abstract).

22 Nakamura, Y. (1989). "A method for dynamic characteristics estimation of subsurface using microtremor on the ground surface". QR of R.T.R.. 30(1). 25-33.

23 Pelli, F., Mangini, M., Bazzurro, P., Eva, C., Spallarossa, D., Barani, S. (2006). "PSHA in northen Italy accounting for non-linear soil amplification effects and epistemic uncertainty". Proc. of 1st ECEES European Conference on Earthquake Engineering and Seismology, Geneva, Switzerland. N:1464.

24 ISTAT (1991). 13th General Census of the Population 1991. Data on the structural characteristics, of the population and housing. Rome.

25 Bramerini, F., Di Pasquale, G., Orsini, A., Pugliese, A., Romeo, R., Sabetta, F., (1995). Seismic Risk in the Italian territory. Technical Report from Servizio Sismico Nazionale SSN/RT/95/01. Roma. (In Italian).

26 Lagomarsino, S. and Giovinazzi, S. (2006). "Macroseismic and Mechanical Models for the Vulnerability assessment of current buildings". Bulletin of Earthquake Engineering, Special Issue "Risk-Ue Project”. 4(4). 